\title{
LINEAR PRESERVERS AND QUANTUM INFORMATION SCIENCE
}

\author{
AJDA FOŠNER, ZEJUN HAUNG, CHI-KWONG LI, AND NUNG-SING SZE
}

Dedicated to Professor Pjek-Hwee Lee on the occasion of his retirement.

\begin{abstract}
In this paper, a brief survey of recent results on linear preserver problems and quantum information science is given. In addition, characterization is obtained for linear operators $\phi$ on $m n \times m n$ Hermitian matrices such that $\phi(A \otimes B)$ and $A \otimes B$ have the same spectrum for any $m \times m$ Hermitian $A$ and $n \times n$ Hermitian $B$. Such a map has the form $A \otimes B \mapsto U\left(\varphi_{1}(A) \otimes \varphi_{2}(B)\right) U^{*}$ for $m n \times m n$ Hermitian matrices in tensor form $A \otimes B$, where $U$ is a unitary matrix, and for $j \in\{1,2\}$, $\varphi_{j}$ is the identity map $X \mapsto X$ or the transposition map $X \mapsto X^{t}$. The structure of linear maps leaving invariant the spectral radius of matrices in tensor form $A \otimes B$ is also obtained. The results are connected bipartite (quantum) systems and are extended to multipartite systems.
\end{abstract}

2010 Math. Subj. Class.: 15A69, 15A86, 15B57, 15A18.

Keywords: Hermitian matrix, linear preserver, spectrum, spectral radius, tensor state.

\section{INTRODUCTION}

The study of linear preserver problems has a long history. It concerns the characterization of linear maps on matrices or operators with special properties. For example, Frobenius [6] showed that a linear operator $\phi: M_{n} \rightarrow M_{n}$ satisfies

$$
\operatorname{det}(\phi(A))=\operatorname{det}(A) \quad \text { for all } A \in M_{n}
$$

if and only if there are $M, N \in M_{n}$ with $\operatorname{det}(M N)=1$ such that $\phi$ has the form

$$
A \mapsto M A N \quad \text { or } \quad A \mapsto M A^{t} N,
$$

where $M_{n}$ denotes the set of $n \times n$ complex matrices. Clearly, a map of the form (10) is linear and leaves the determinant function invariant. It is interesting that a linear map preserving the determinant function must be of this form. In [4 Dieudonné showed that an invertible linear operator $\phi: M_{n} \rightarrow M_{n}$ maps the set of singular matrices into itself if and only if there are invertible $M, N \in M_{n}$ such that $\phi$ has the form (10). One may see [15] and its references for results on linear preserver problems. There are many new directions and active research on preserver problems motivated by theory and applications; see [1, 26, 33].

In this paper, we focus on linear preserver problems related to quantum information science. In Section 2, we briefly survey some recent results on such research, and motivate our study in Section 3, in which we characterize linear preservers of the spectral radius or the spectrum of the tensor product of two Hermitian matrices, and discuss the implications of the result to bipartite quantum systems. The results are extended to the tensor product of $m$ Hermitian matrices with $m>2$ corresponding to the multipartite quantum systems. Additional remarks, results and open problems are also presented. 


\section{Quantum Information SCIENCE AND PRESERVERS}

Let $H_{n}$ be the set of Hermitian matrices in $M_{n}$. In quantum physics, quantum states of a system with $n$ physical states are represented as density matrices $A$ in $H_{n}$, i.e., $A$ is positive semi-definite with trace one. Rank one orthogonal projections are pure states.

The classical Wigner's theorem in quantum mechanics asserts that a bijective map $\phi$ on the set of pure states satisfying $\operatorname{tr}(A B)=\operatorname{tr}(\phi(A) \phi(B))$ must be of the form

$$
A \mapsto U A U^{*} \quad \text { or } \quad A \mapsto U A^{t} U^{*}
$$

for some unitary operator $U$. Uhlhorn [32] showed that a bijective map $\phi$ on the set of pure states also has the form (2) under the weaker assumption that $\operatorname{tr}(A B)=0$ if and only if $\operatorname{tr} \phi(A) \phi(B))=0$. The result was extended to Hilbert modules over matrix algebras, prime $\mathrm{C}^{*}$-algebras, and indefinite inner product spaces; see [21, 24]. In [16], the authors extended Uhlhorn's result to Hermitian matrices, symmetric matrices, the set of orthogonal projections, the set of rank one orthogonal projections, and the set of effect algebra, and studied bijective maps on these matrix sets such that

$$
\operatorname{tr}(A B)=c \quad \text { if and only if } \quad \operatorname{tr}(\phi(A) \phi(B))=c
$$

for a given $c>0$.

In a series of interesting papers [22, 23, 24, 25, 27, Molnár and his collaborators characterized bijective maps on the set of complex matrices, Hermitian matrices, bounded observables, effect algebra, etc. preserving special subsets or relations. In many cases, the map has the form (2). One may see also [26] for additional results along this direction.

Suppose $A \in H_{m}$ and $B \in H_{n}$ are the states of two quantum systems. Then the tensor (Kronecker) state $A \otimes B \in H_{m n}$ describes the joint (bipartite) system. A density matrix $C \in H_{m n}$ is separable if it is the convex combination of tensor states, i.e., $C=\sum_{j=1}^{r} t_{j} A_{j} \otimes B_{j}$ for some positive numbers $t_{1}, \ldots, t_{r}$ summing up to one, and tensor states $A_{1} \otimes B_{1}, \ldots, A_{r} \otimes B_{r}$. Otherwise, $C$ is entangled. Identifying separable states in $H_{m n}$ is an NP-hard problem; see [7]. Nevertheless, there is of interest in finding easy ways to check necessary or sufficient conditions of separability of states. In particular, it is interesting to find transformations which will simplify a given state so that it is easier to determine whether it is separable or not. Evidently, the transformations used should not change the set of separable states. This leads to the study of linear operators leaving invariant the set of separable states (entangled states). Similar definitions and questions can be considered for multipartite systems. The following result was proved in [5].

Theorem 2.1. Let $n_{1}, \ldots, n_{m} \in\{2,3, \ldots\}$ and $N=\prod_{j=1}^{m} n_{j}$. Suppose $\mathcal{S}$ is one of the following.

(a) The set of tensor product (of pure) states $A_{1} \otimes \cdots \otimes A_{m}$, where $A_{j} \in H_{n_{j}}$ is a (pure) state for each $j \in\{1, \ldots, m\}$.

(b) The set of separable states in $H_{N}$, viz, the convex hull of the set of tensor product (of pure) states.

Then a linear map $\phi: H_{N} \rightarrow H_{N}$ satisfies $\phi(\mathcal{S})=\mathcal{S}$ if and only if there is a permutation $\left(p_{1}, \ldots, p_{m}\right)$ of $(1, \ldots, m)$ such that

$$
A_{1} \otimes \cdots \otimes A_{m} \mapsto \psi_{1}\left(A_{p_{1}}\right) \otimes \cdots \otimes \psi_{m}\left(A_{p_{m}}\right),
$$


where for each $j \in\{1, \ldots, m\}, n_{j}=n_{p_{j}}$ and $\psi_{j}: M_{n_{j}} \rightarrow M_{n_{j}}$ is a linear map of the form

$$
X \mapsto U_{j} X U_{j}^{*} \quad \text { or } \quad X \mapsto U_{j} X^{t} U_{j}^{*}
$$

for a unitary $U_{j} \in M_{n_{j}}$.

The result was generalized in three directions by researchers. First, Hou and his associates [8] extended the result to the infinite dimensional setting and characterized bounded invertible linear maps leaving invariant the set of tensor product of rank one orthogonal projections acting on infinite dimensional Hilbert spaces, or its convex hull, i.e., the set of separable states. Second, Lim [18] characterized linear map $\phi: H_{n_{1}} \otimes \cdots \otimes H_{n_{m}} \rightarrow H_{\tilde{n}_{1}} \otimes \cdots \otimes H_{\tilde{n}_{m}}$ such that $\phi$ maps the set of tensor (separable) states in the domain into the set of tensor (separable) states in the codomain. Third, the authors in [17] characterize linear map $\phi: H_{n_{1}} \otimes \cdots \otimes H_{n_{m}} \rightarrow H_{n_{1}} \otimes \cdots \otimes H_{n_{m}}$ such that $\phi\left(\mathcal{S}_{1}\right)=\mathcal{S}_{2}$, where

$$
\mathcal{S}_{1}=\left\{X_{1} \otimes \cdots \otimes X_{m}: X_{j} \in \mathcal{U}\left(C_{j}\right), j=1, \ldots, m\right\}
$$

and

$$
\mathcal{S}_{2}=\left\{Y_{1} \otimes \cdots \otimes Y_{m}: Y_{j} \in \mathcal{U}\left(D_{j}\right), j=1, \ldots, m\right\}
$$

for given states $C_{j}, D_{j} \in H_{n_{j}}$ with $j=1, \ldots, m$ and

$$
\mathcal{U}(X)=\left\{U^{*} X U: U \text { unitary }\right\}
$$

is the unitary (similarity) orbit of $X$. When $C_{i}$ and $D_{i}$ are pure states, the study reduces to the problem treated in [5], and reveals the fact that there are linear transformations converting a unitary orbit to a different unitary orbit.

In [10], the author showed a number of interesting linear preserver results related to quantum information science. A vector state of a quantum system with $m$ measurable physical states can be represented as a unit vector $u$ in $\mathbb{C}^{m}$. A product state of two vector states $u \in \mathbb{C}^{m}$ and $v \in \mathbb{C}^{n}$ is the tensor product $u \otimes v \in \mathbb{C}^{m n}$, and unit vectors in $\mathbb{C}^{m n}$ can be viewed as vector states in the bipartite system with $\mathbb{C}^{m}$ and $\mathbb{C}^{n}$ as components. Every vector $w \in \mathbb{C}^{m n}$ can be identified with an $m \times n$ matrix $[w]$ by putting the first $n$ entries in the first row, the next $n$ entries in the second row, etc. In particular, $u \otimes v$ can be identify with the matrix $u v^{t}$. The singular value decomposition of the matrix $[w]=\sum_{j=1}^{k} s_{j} u_{j} v_{j}^{t}$ corresponds to the Schmidt decomposition $w=\sum_{j=1}^{k} s_{j} u_{j} \otimes v_{j}$. The Schmidt rank of a vector (state) $w$ is the rank of the matrix $[w]$. Clearly, the linear span of product states $u \otimes v$ will generate all the vectors in $\mathbb{C}^{m n}$, and a linear map $L$ on $\mathbb{C}^{m n}$ is completely determined once we know $L(u \otimes v$ ) for all (or $m n$ linearly independent) product states $u \otimes v$. In [10], the author used some classical results on linear preservers to study maps preserving $\mathcal{P}_{k}$, the set of all states with Schmidt rank at most $k$ for a given $k \leq \min \{m, n\}$. In particular, it was shown that an invertible linear map $L: \mathbb{C}^{m n} \rightarrow \mathbb{C}^{m n}$ satisfies $L\left(\mathcal{P}_{k}\right) \subseteq \mathcal{P}_{k}$ if and only if there are unitary matrices $P \in M_{m}$ and $Q \in M_{n}$ such that one of the following holds.

(a) $L(u \otimes v)=P u \otimes Q v$ for all $(u, v) \in \mathbb{C}^{m} \times \mathbb{C}^{n}$.

(b) $m=n$ and $L(u \otimes v)=Q v \otimes P u$ for all $(u, v) \in \mathbb{C}^{m} \times \mathbb{C}^{n}$.

Suppose $\mathcal{S}_{k}$ is the set of all vectors $w \in \mathbb{C}^{m n}$ with Schmidt rank at most $k$. Then an invertible linear map $L: \mathbb{C}^{m n} \rightarrow \mathbb{C}^{m n}$ satisfies $L\left(\mathcal{S}_{k}\right) \subseteq \mathcal{S}_{k}$ if and only if there are invertible matrices $P \in M_{m}$ and $Q \in M_{n}$ such that (a) or (b) holds. 
Another result in [10] asserts that an invertible linear map $\Phi: M_{m n} \rightarrow M_{m n}$ satisfies $\Phi(\mathcal{S}) \subseteq \mathcal{S}$, where $\mathcal{S}$ is the set of rank one matrices of the form $u v^{t}$ such that $u$ and $v$ have Schmidt rank at most $k$ if and only if $\Phi$ is a composition of one or more of the following maps.

(1) The transpose map $X \mapsto X^{t}$.

(2) $X \mapsto\left(P_{1} \otimes Q_{1}\right) X\left(P_{2} \otimes Q_{2}\right)$ for some invertible matrices $P_{i} \in M_{m}$ and $Q_{i} \in M_{n}$ for $i=1,2$.

(3) $k=1$, the partial transpose map $\left[X_{i j}\right]_{1 \leq i, j \leq m} \mapsto\left[X_{i j}^{t}\right]_{1 \leq i, j \leq m}$, where $X_{i j} \in M_{n}$.

Furthermore, Johnston considered the norm on $\mathbb{C}^{m n}$ defined by

$$
\|u\|_{k}=\max \left\{\left|v^{*} u\right|: v \in \mathbb{C}^{m n}, v^{*} v=1, \operatorname{rank}([v]) \leq k\right\}=\left\{\sum_{j=1}^{k} s_{j}^{2}\right\}^{1 / 2},
$$

where $s_{1} \geq s_{2} \geq \cdots$ are the singular values of $[u]$, for any $k \leq \min \{m, n\}$. He also considered the norm on $M_{m n}$ defined by

$$
\||| C||_{k}=\max \left\{\left|u^{*} C v\right|: u, v \in \mathbb{C}^{m n}, u^{*} u=v^{*} v=1, \operatorname{rank}([u]) \leq k, \operatorname{rank}([v]) \leq k\right\} .
$$

These norms have recently been studied in [3, 11, 12, 13, 28] and were shown to be related to the problem of characterizing $k$-positive linear maps and detecting bound entangled non-positive partial transpose states.

In connection to the preserver problems, it was shown that a linear map $L: \mathbb{C}^{m n} \rightarrow \mathbb{C}^{m n}$ satisfies

$$
\|L(u)\|_{k}=\|u\|_{k} \quad \text { for all } u \in \mathbb{C}^{m n}
$$

if and only if there are unitary $P \in M_{m}$ and $Q \in M_{n}$ such that condition (a) or (b) mentioned above holds.

If $k=\min \{m, n\}$ one sees that $\|C\|_{k}$ is just the operator norm. It is known that a linear preserver on $M_{m n}$ of the operator norm has the form

$$
X \mapsto U X V \quad \text { or } \quad X \mapsto U X^{t} V
$$

for some unitary $U, V \in M_{m n}$. For $k<\min \{m, n\}$, Johnston showed that a linear map $\Phi: M_{m n} \rightarrow$ $M_{m n}$ satisfies

$$
\||\Phi(X)|\|_{k}=\|\| X \mid \|_{k} \quad \text { for all } X \in M_{m n}
$$

if and only if $\Phi$ is a composition of one or more of the maps described in (1), (2) or (3) above with the additional restriction that $P$ and $Q$ in (2) are unitary.

Many of the above results are extended to multi-partite system, e.g., [5, 10, 17, 18].

Next, we consider another line of research in preserver problems. There has been considerable interest in studying spectrum preserving maps (see [2, 9, 19] etc). On Hermitian matrices, it is known that a linear map on $H_{n}$ that leaves invariant the spectrum has the form

$$
A \mapsto U A U^{*} \quad \text { or } \quad A \mapsto U A^{t} U^{*}
$$

for some unitary $U \in M_{n}$. If one gives up the Hermitian preserving property and considers a (complex) linear operator $\phi: M_{n} \rightarrow M_{n}$ that leaves invariant the eigenvalues of Hermitian matrices, then $\phi$ has the form

$$
A \mapsto S A S^{-1} \quad \text { or } \quad A \mapsto S A^{t} S^{-1}
$$


for some invertible $S \in M_{n}$.

In [30, 31, the authors studied non-classical correlation in a bipartite systems and showed that for any spectrum preserving linear map $\phi: H_{n} \rightarrow M_{n}$, either

$$
\sigma\left(\left(\operatorname{Id}_{m} \otimes \phi\right)(C)\right)=\sigma(C) \quad \text { for all } \quad C \in H_{m} \otimes H_{n},
$$

or

$$
\sigma\left(\left(\operatorname{Id}_{m} \otimes \phi\right)(C)=\sigma\left(\mathrm{PT}_{2}(C)\right) \quad \text { for all } \quad C \in H_{m} \otimes H_{n},\right.
$$

where $\mathrm{PT}_{2}(A \otimes B)=A \otimes B^{t}$ is the partial transpose map for the second component and $\mathrm{Id}_{m}$ is the identity map on $m \times m$ matrices.

Following this line of study, we consider linear operators leaving invariant the spectrum of tensor states and related problems in the next section. It turns out that even if one assumes only that a linear operator $\phi$ leaves invariant the spectrum of matrices in tensor form $A \otimes B \in H_{m} \otimes H_{n}$, the operator $\phi$ has a nice structure, namely, up to a unitary similarity, $\phi$ has the form $A \otimes B \mapsto$ $\psi_{1}(A) \otimes \psi_{2}(B)$ for all tensor states $A \otimes B$, where $\psi_{j}$ is the identity map $X \mapsto X$ or the transposition map $X \mapsto X^{t}$. Moreover, if $\sigma(C)=\sigma(\phi(C))$ for a carefully chosen $C \in H_{m n}$, then $\phi$ will actually preserve the spectrum of every matrix in $H_{m n}$, and will be of the form $X \mapsto V X V^{*}$ or $X \mapsto V X^{t} V^{*}$ on $H_{m n}$ for some unitary matrix $V \in H_{m n}$. Similar results are obtained for linear maps leaving invariant the spectral radius of tensor states $A \otimes B$ in $H_{m} \otimes H_{n}$.

\section{Preservers of SPECTRAl RAdius or SPECTRUm}

Suppose $A \in H_{m}$ has eigenvalues $a_{1} \geq \cdots \geq a_{m}$ associated with orthonormal eigenvectors $x_{1}, \ldots, x_{m}$, and $B \in H_{n}$ has eigenvalues $b_{1} \geq \cdots \geq b_{n}$ associated with orthonormal eigenvectors $y_{1}, \ldots, y_{n}$, then $A \otimes B$ has eigenvalues $a_{r} b_{s}$ associated with eigenvectors $x_{r} \otimes y_{s}$ for $(r, s) \in$ $\{1, \ldots, m\} \times\{1, \ldots, n\}$. Denote by $\sigma(X)$ and $r(X)$ the spectrum and spectral radius of a matrix $X \in M_{n}$. In Subsection 3.1, we show that a linear map $\phi: H_{m} \otimes H_{n} \rightarrow H_{m} \otimes H_{n}$ satisfies

$$
\sigma(\phi(A \otimes B))=\sigma(A \otimes B)
$$

for all $A \otimes B \in H_{m} \otimes H_{n}$ if and only if there is a unitary $U \in M_{m n}$ such that

$$
A \otimes B \mapsto U\left(\varphi_{1}(A) \otimes \varphi_{2}(B)\right) U^{*},
$$

where $\varphi_{j}, j=1,2$, is either the identity map or the transposition map $X \mapsto X^{t}$ (see Theorem 3.2). Furthermore, we will also show that a linear map on $H_{m n}$ leaving the spectral radius of tensor states invariant, i.e.,

$$
r(\phi(A \otimes B))=r(A \otimes B)
$$

for all $A \otimes B \in H_{m} \otimes H_{n}$, is \pm 1 multiple of a map of the standard form (4) (see Theorem [3.3). In Subsection 3.2, we will extend the results to multipartite systems (Theorem 3.4 and Theorem 3.5). Additional remarks, results, and open problems will be presented in Subsection 3.3.

3.1. Bipartite system. Throughout this paper, we denote by $E_{i j}, 1 \leq i, j \leq n$ the standard basis of $M_{n}$. We need the following lemma.

Lemma 3.1. Let $m>n$ and $A \in H_{m}$ with $\sigma(A)=\left\{a_{1}, \ldots, a_{n}, 0, \ldots, 0\right\}$. If

$$
\sigma\left(A+t\left(I_{n} \oplus 0_{m-n}\right)\right)=\left\{a_{1}+t, \ldots, a_{n}+t, 0, \ldots, 0\right\} \text { for all } t \in \mathbb{R},
$$


then $A=B \oplus 0_{m-n}$ for some $B \in H_{n}$.

Proof. Choose a sufficient large $s \in \mathbb{R}$ so that $C=A+s\left(I_{n} \oplus 0_{m-n}\right)$ is positive semi-definite with eigenvalues $c_{1}, \ldots, c_{n}, 0, \ldots, 0$ where $c_{j}=a_{j}+s, j=1, \ldots, n$. Then

$$
\sigma\left(C+t\left(I_{n} \oplus 0_{m-n}\right)\right)=\sigma\left(A+(s+t)\left(I_{n} \oplus 0_{m-n}\right)\right)=\left\{c_{1}+t, \ldots, c_{n}+t, 0, \ldots, 0\right\} .
$$

Denote by $\left\{e_{1}, \ldots, e_{m}\right\}$ the standard basis of $\mathbb{C}^{m}$. Then for any unit vector $v \in \operatorname{span}\left\{e_{n+1}, \ldots, e_{m}\right\}$,

$$
v^{*} C v=v^{*}\left(C+t\left(I_{n} \oplus 0_{m-n}\right)\right) v \in \operatorname{conv}\left\{c_{1}+t, \ldots, c_{n}+t, 0\right\} \quad \text { for all } \quad t \in \mathbb{R},
$$

where conv $S$ denote the convex hull of the set $S$. Since this holds for all $t$ in $\mathbb{R}$, this is possible only when $v^{*} C v=0$. As $C$ is positive semi-definite, $v$ is an eigenvector of $C$ with eigenvalue 0 . As $v$ is arbitrary in $\operatorname{span}\left\{e_{n+1}, \ldots, e_{m}\right\}, C$ must have the form $C_{1} \oplus 0_{m-n}$. Hence, $A=B \oplus 0_{m-n}$ with $B=C_{1}-s I_{n}$.

Theorem 3.2. A linear map $\phi: H_{m n} \rightarrow H_{m n}$ satisfies

$$
\sigma(\phi(A \otimes B))=\sigma(A \otimes B)
$$

for all $A \otimes B \in H_{m} \otimes H_{n}$ if and only if there is a unitary $U \in M_{m n}$ such that

$$
\phi(A \otimes B)=U\left(\varphi_{1}(A) \otimes \varphi_{2}(B)\right) U^{*},
$$

where $\varphi_{j}$ is the identity map or the transposition map $X \mapsto X^{t}$ for $j \in\{1,2\}$.

Proof. The sufficiency part is clear. We consider the necessity part. Since $\sigma\left(\phi\left(I_{m} \otimes I_{n}\right)\right)=\sigma\left(I_{m} \otimes\right.$ $\left.I_{n}\right)=\{1\}$, we see that $\phi\left(I_{m} \otimes I_{n}\right)=I_{m} \otimes I_{n}$. Consider any distinct pairs $(j, k)$ and $(r, s)$ for $j, r \in\{1, \ldots, m\}, k, s \in\{1, \ldots, n\}$. Then $\phi\left(E_{j j} \otimes E_{k k}\right)$ and $\phi\left(E_{r r} \otimes E_{s s}\right)$ are nonzero orthogonal projections. Now, $I_{m n}=\phi\left(I_{m n}\right)=\sum_{j, k} \phi\left(E_{j j} \otimes E_{k k}\right)$ has trace $m n$. It follows that each $\phi\left(E_{j j} \otimes E_{k k}\right)$ has rank one. Moreover, $\phi\left(E_{j j} \otimes E_{k k}\right)$ and $\phi\left(E_{r r} \otimes E_{s s}\right)$ have disjoint range spaces for any distinct pairs $(j, k)$ and $(r, s)$. Hence, there exists a unitary $W \in M_{m n}$ such that

$$
\phi\left(E_{j j} \otimes E_{k k}\right)=W\left(E_{j j} \otimes E_{k k}\right) W^{*}
$$

for all $1 \leq j \leq m$ and $1 \leq k \leq n$.

For any $B \in H_{n}, t \in \mathbb{R}$, and $1 \leq j \leq m$, we have

$$
\begin{aligned}
& \sigma\left(\phi\left(E_{j j} \otimes B\right)+t \phi\left(E_{j j} \otimes I_{n}\right)\right)=\sigma\left(\phi\left(E_{j j} \otimes\left(B+t I_{n}\right)\right)\right) \\
= & \sigma\left(E_{j j} \otimes\left(B+t I_{n}\right)\right)=\{b+t: b \in \sigma(B)\} \cup\{0\} .
\end{aligned}
$$

Since $\phi\left(E_{j j} \otimes I_{n}\right)=W\left(E_{j j} \otimes I_{n}\right) W^{*}$, applying Lemma 3.1 and using permutation similarity if necessary, we have

$$
\phi\left(E_{j j} \otimes B\right)=W\left(E_{j j} \otimes \psi_{j}(B)\right) W^{*}
$$

for some $\psi_{j}(B) \in H_{n}$. Furthermore, $B$ and $\psi_{j}(B)$ have the same spectrum. So $\psi_{j}$ has the form

$$
B \mapsto U_{j} B U_{j}^{*} \quad \text { or } \quad B \mapsto U_{j} B^{t} U_{j}^{*}
$$

for some unitary $U_{j}$. Replace $W$ with $W\left(U_{1} \oplus \cdots \oplus U_{m}\right)$. Then

$$
\phi\left(E_{j j} \otimes B\right)=W\left(E_{j j} \otimes \varphi_{j}(B)\right) W^{*}
$$

for all $1 \leq j \leq m$ and $B \in H_{n}$, where each map $\varphi_{j}$ is the identity map or the transposition map $X \mapsto X^{t}$. 
Repeating the same argument, one can show that for any unitary $U \in M_{m}$,

$$
\phi\left(U E_{j j} U^{*} \otimes B\right)=W_{U}\left(E_{j j} \otimes \varphi_{j, U}(B)\right) W_{U}^{*}
$$

for all $1 \leq j \leq m$ and $B \in H_{n}$, where $W_{U} \in M_{m n}$ is a unitary matrix, depending on $U$, and $\varphi_{j, U}$ is either the identity map or the transposition map, depending on $j$ and $U$. Replacing $\phi$ by the map $A \mapsto W_{I_{m n}}^{*} \phi(A) W_{I_{m n}}$, we may assume that

$$
W_{I_{m n}}=I_{m n} \quad \text { and } \quad \phi\left(E_{j j} \otimes E_{k k}\right)=E_{j j} \otimes E_{k k}
$$

for all $1 \leq j \leq m$ and $1 \leq k \leq n$. Now, for any real symmetric $S \in H_{n}$ and unitary $U \in M_{m}$, we have $\varphi_{j, U}(S)=S$ for all $j=1, \ldots, m$, and, hence,

$$
\phi\left(I_{m} \otimes S\right)=\phi\left(\sum_{j=1}^{m} U E_{j j} U^{*} \otimes S\right)=W_{U}\left(\sum_{j=1}^{m} E_{j j} \otimes S\right) W_{U}^{*}=W_{U}\left(I_{m} \otimes S\right) W_{U}^{*}
$$

for some unitary $W_{U} \in M_{m n}$. In particular, when $U=I_{m}, \phi\left(I_{m} \otimes S\right)=I_{m} \otimes S$. Thus, $W_{U}\left(I_{m} \otimes S\right) W_{U}^{*}=I_{m} \otimes S$. It follows that $W_{U}$ commutes with $I_{m} \otimes S$ for all real symmetric $S$. Hence, $W_{U}$ has the form $V_{U} \otimes I_{n}$ for some $V_{U} \in M_{m}$ and

$$
\phi\left(U E_{j j} U^{*} \otimes B\right)=\left(V_{U} E_{j j} V_{U}^{*}\right) \otimes \varphi_{j, U}(B)
$$

for $1 \leq j \leq m$ and $B \in M_{m}$. Consider the linear maps $\operatorname{tr}_{1}: H_{m n} \rightarrow H_{n}$ and $\Phi: H_{m n} \rightarrow H_{n}$ defined by

$$
\operatorname{tr}_{1}(A \otimes B)=(\operatorname{tr} A) B \quad \text { and } \quad \Phi(A \otimes B)=\operatorname{tr}_{1}(\phi(A \otimes B))
$$

for any $A \otimes B \in H_{m} \otimes H_{n}$. Then

$$
\Phi\left(U E_{j j} U^{*} \otimes B\right)=\varphi_{j, U}(B) .
$$

Recall that a continuous image of a connected space is still connected. Since $\Phi$ is linear and continuous, $\left\{x x^{*} \in M_{m}: x^{*} x=1\right\}$ is connected, and $\varphi_{j, U}$ is either the identity map or the transposition map, all the maps $\varphi_{j, U}$ have to be the same. Replacing $\phi$ by the map $A \otimes B \mapsto$ $\phi\left(A \otimes B^{t}\right)$, if necessary, we may assume that this common map is the identity map. Next, by linearity, one can conclude that for every $A \in H_{m}$ and $B \in H_{n}$ we have

$$
\phi(A \otimes B)=\varphi_{1}(A) \otimes B
$$

for some $\varphi_{1}(A) \in H_{m}$, where $\varphi_{1}(A)$ depends on $A$ only. Note that $\varphi_{1}: H_{m} \rightarrow H_{m}$ is a linear map and $\sigma\left(\varphi_{1}(A)\right)=\sigma(A)$ for all $A \in H_{m}$. Hence, by [19], a map $\varphi_{1}$ has the form $A \mapsto V A V^{*}$ or $A \mapsto V A^{t} V^{*}$. The proof is completed.

In the following, we consider linear maps on $H_{m n}$ leaving the spectral radius invariant.

Theorem 3.3. A linear map $\phi: H_{m n} \rightarrow H_{m n}$ satisfies

$$
r(\phi(A \otimes B))=r(A \otimes B)
$$

for all $A \otimes B \in H_{m} \otimes H_{n}$ if and only if there is a unitary $U \in M_{m n}$ and $\lambda \in\{-1,1\}$ such that

$$
\phi(A \otimes B)=\lambda U\left(\varphi_{1}(A) \otimes \varphi_{2}(B)\right) U^{*},
$$

where $\varphi_{j}$ is the identity map or the transposition map $X \mapsto X^{t}$ for $j \in\{1,2\}$. 
Proof. The sufficiency part is clear. For the converse, suppose that a linear map $\phi: H_{m n} \rightarrow H_{m n}$ preserves the spectral radius of tensor states and let $1 \leq j \leq m, 1 \leq k \leq n$. Then $\phi\left(E_{j j} \otimes E_{k k}\right)$ has an eigenvalue in $\{1,-1\}$. For $t \neq k$, we have $r\left(\phi\left(E_{j j} \otimes\left(E_{k k} \pm E_{t t}\right)\right)\right)=1$. This yields that every eigenvector of $\phi\left(E_{j j} \otimes E_{k k}\right)$ corresponding to the eigenvalue 1 or -1 lies in the kernel of $\phi\left(E_{j j} \otimes E_{t t}\right)$. Since this is true for any pair of $k$ and $t$, for any orthogonal diagonal matrix $D \in M_{n}$ at least $n$ eigenvalues of $\phi\left(E_{j j} \otimes D\right)$ lie in $\{1,-1\}$. Since $r\left(\phi\left(\left(E_{j j} \pm E_{s s}\right) \otimes D\right)\right)=1$ for any $j \neq s$, $1 \leq j, s \leq m$, and any diagonal orthogonal matrix $D \in H_{n}, \phi\left(E_{j j} \otimes D\right)$ and $\phi\left(E_{s s} \otimes D\right)$ have disjoint support and, hence, $\phi\left(E_{j j} \otimes D\right)$ has rank $n$. It follows that all $\phi\left(E_{j j} \otimes E_{k k}\right)$ must be rank one and $\phi\left(E_{j j} \otimes E_{k k}\right)$ and $\phi\left(E_{s s} \otimes E_{t t}\right)$ have disjoint support for any distinct $(j, k)$ and $(s, t)$. Therefore, there is a unitary $W \in M_{m n}$ and $\mu_{j k} \in\{1,-1\}$ such that

$$
\phi\left(E_{j j} \otimes E_{k k}\right)=\mu_{j k} W\left(E_{j j} \otimes E_{k k}\right) W^{*} \quad \text { for all } \quad 1 \leq j \leq m, 1 \leq k \leq n .
$$

For the sake of the simplicity, suppose that $W=I_{m n}$ and $\phi\left(E_{j j} \otimes I_{n}\right)=E_{j j} \otimes P_{j}$, where $P_{1}, \ldots, P_{m} \in$ $H_{n}$ are diagonal orthogonal matrices.

For any unitary $V \in M_{n}$, applying the same arguments to $E_{j j} \otimes V E_{k k} V^{*}, 1 \leq j \leq m, 1 \leq k \leq n$, we see that $\phi\left(E_{j j} \otimes V E_{k k} V^{*}\right)$ has rank one with spectral radius 1 . If $t>0$, we have

$$
r\left(\phi\left(E_{j j} \otimes\left(V E_{k k} V^{*}+t I_{n}\right)\right)\right)=1+t .
$$

Thus, the eigenspace of the nonzero eigenvalue of $\phi\left(E_{j j} \otimes V E_{k k} V^{*}\right)$ must lie in the eigenspace of $\phi\left(E_{j j} \otimes I_{n}\right)=E_{j j} \otimes P_{j}$. Consequently, we see that $\phi\left(E_{j j} \otimes B\right)=E_{j j} \otimes \varphi_{j}(B)$ for any $B \in H_{n}$. Clearly, $\varphi_{j}$ preserves spectral radius on $H_{n}$ and, hence, by [14] it has the form

$$
B \mapsto \xi Y B Y^{*} \quad \text { or } \quad B \mapsto \xi Y B^{t} Y^{*}
$$

for some $\xi \in\{1,-1\}$ and unitary $Y \in M_{n}$. In particular, $\varphi_{j}\left(I_{n}\right) \in\left\{I_{n},-I_{n}\right\}$. So, $\phi\left(I_{m n}\right)=D \otimes I_{n}$ for some diagonal orthogonal matrix $D \in M_{m}$.

By considering $U E_{j j} U^{*} \otimes E_{k k}$ for unitary $U \in M_{m}$ and using the same arugment as in the last paragraph, one can show that $\phi\left(I_{m n}\right)=I_{m} \otimes \tilde{D}$ for some diagonal orthogonal matrix $\tilde{D} \in M_{n}$. Since $\phi\left(I_{m n}\right)=I_{m} \otimes \tilde{D}=D \otimes I_{n}$, we conclude that $\phi\left(I_{m n}\right)= \pm I_{m n}$. Without loss of generality, we may assume that $\phi\left(I_{m n}\right)=I_{m n}$. Thus, all $\mu_{j k}$ are equal to 1, i.e.,

$$
\phi\left(E_{j j} \otimes E_{k k}\right)=E_{j j} \otimes E_{k k} \quad \text { for all } 1 \leq j \leq m, 1 \leq k \leq n .
$$

For any $A \otimes B \in H_{m} \otimes H_{n}$, there are unitary $U \in M_{m}$ and $V \in M_{n}$ such that $U A U^{*}$ and $V B V^{*}$ are diagonal matrices. Without loss of generality, we assume that $A=\operatorname{Diag}\left(a_{1}, \ldots, a_{m}\right)$ and $B=\operatorname{Diag}\left(b_{1}, \ldots, b_{n}\right)$. Then

$$
\phi(A \otimes B)=\phi\left(\left(\sum_{j=1}^{m} a_{j} E_{j j}\right) \otimes\left(\sum_{k=1}^{n} b_{k} E_{k k}\right)\right)=A \otimes B .
$$

Thus, $\sigma(\phi(A \otimes B))=\sigma(A \otimes B)$ and the result is followed by Theorem 3.2 .

3.2. Multipartite systems. In this section we will extend Theorem 3.2 and Theorem 3.3 to multipartite system $H_{n_{1} \cdots n_{m}}=H_{n_{1}} \otimes \cdots \otimes H_{n_{m}}, m \geq 2$. 
Theorem 3.4. A linear map $\phi: H_{n_{1} \cdots n_{m}} \rightarrow H_{n_{1} \cdots n_{m}}$ satisfies

$$
\sigma\left(\phi\left(A_{1} \otimes \cdots \otimes A_{m}\right)\right)=\sigma\left(A_{1} \otimes \cdots \otimes A_{m}\right)
$$

for all $A_{1} \otimes \cdots \otimes A_{m} \in H_{n_{1} \cdots n_{m}}$ if and only if there is a unitary $U \in M_{n_{1} \cdots n_{m}}$ such that

$$
\phi\left(A_{1} \otimes \cdots \otimes A_{m}\right)=U\left(\varphi_{1}\left(A_{1}\right) \otimes \cdots \otimes \varphi_{m}\left(A_{m}\right)\right) U^{*},
$$

where $\varphi_{j}$ is the identity map or the transposition map $X \mapsto X^{t}$ for $j \in\{1, \ldots, m\}$.

Proof. The sufficiency part is clear. To prove the necessity part, we use induction on $m$. By Theorem 3.2, we already know that the statement of Theorem 3.4 is true for bipartite systems. So, assume that $m \geq 3$ and that the result holds for all $(m-1)$-partite systems. We would like to prove that the same is true for $m$-partite systems.

As in the proof of Theorem 3.2, we can show that there exists a unitary $W \in M_{n_{1} \cdots n_{m}}$ such that

$$
\phi\left(E_{j_{1} j_{1}} \otimes \cdots \otimes E_{j_{m} j_{m}}\right)=W\left(E_{j_{1} j_{1}} \otimes \cdots \otimes E_{j_{m} j_{m}}\right) W^{*}
$$

for all $1 \leq j_{p} \leq n_{p}$ with $1 \leq p \leq m$. Moreover, for any $B \in H_{n_{1}}$ and $1 \leq j_{p} \leq n_{p}$ with $2 \leq p \leq m$, we have

$$
\phi\left(B \otimes E_{j_{2} j_{2}} \otimes \cdots \otimes E_{j_{m} j_{m}}\right)=W\left(\psi_{j_{2}, \ldots, j_{m}}(B) \otimes E_{j_{2} j_{2}} \otimes \cdots \otimes E_{j_{m} j_{m}}\right) W^{*}
$$

for some $\varphi_{j_{2}, \ldots, j_{m}}(B) \in H_{n_{1}}$. Then $B$ and $\varphi_{j_{2}, \ldots, j_{m}}(B)$ have the same spectrum. By the fact that $\varphi_{j_{2}, \ldots, j_{m}}\left(E_{k k}\right)=E_{k k}$ for all $1 \leq k \leq n_{1}$, the map $\varphi_{j_{2}, \ldots, j_{m}}$ can be assumed either the identity map or the transposition map. By a similar argument, we can show that

$$
\phi\left(B \otimes\left(\bigotimes_{p=2}^{m} U_{p} E_{j_{p} j_{p}} U_{p}^{*}\right)\right)=W_{U_{2}, \ldots, U_{m}}\left(\varphi_{j_{2}, \ldots, j_{m}}^{U_{2}, \ldots, U_{m}}(B) \otimes E_{j_{2} j_{2}} \otimes \cdots \otimes E_{j_{m} j_{m}}\right) W_{U_{2}, \ldots, U_{m}}^{*}
$$

for all $B \in H_{n_{1}}$ and $1 \leq j_{p} \leq n_{p}$ with $2 \leq p \leq m$, where $W_{U_{2}, \ldots, U_{m}} \in M_{n_{1} \cdots n_{m}}$ is a unitary matrix depending on $U_{2}, \ldots, U_{m}$ only and $\varphi_{j_{2}, \ldots, j_{m}}^{U_{2}, \ldots, U_{m}}$ is either the identity map or the transposition map, depending on $j_{2}, \ldots, j_{m}$ and $U_{2}, \ldots, U_{m}$. Replacing $\phi$ by the map $A \mapsto W_{I_{n_{2}}, \ldots, I_{n_{m}}}^{*} \phi(A) W_{I_{n_{2}}, \ldots, I_{n_{m}}}$, we may assume that

$$
W_{I_{n_{2}}, \ldots, I_{n_{m}}}=I_{n_{1} \cdots n_{m}} \quad \text { and } \quad \phi\left(E_{j_{1} j_{1}} \otimes \cdots \otimes E_{j_{m} j_{m}}\right)=E_{j_{1} j_{1}} \otimes \cdots \otimes E_{j_{m} j_{m}}
$$

for all $1 \leq j_{p} \leq n_{p}$ with $1 \leq p \leq m$. Again, considering all symmetric $S \in H_{n_{1}}$ as in the proof of Theorem 3.2, we can show that there exists $V_{U_{2}, \ldots, U_{m}} \in M_{n_{2} \cdots n_{m}}$ such that

$$
\phi\left(B \otimes\left(\bigotimes_{p=2}^{m} U_{p} E_{j_{p} j_{p}} U_{p}^{*}\right)\right)=\varphi_{j_{2}, \ldots, j_{m}}^{U_{2}, \ldots, U_{m}}(B) \otimes V_{U_{2}, \ldots, U_{m}}\left(E_{j_{2} j_{2}} \otimes \cdots \otimes E_{j_{m} j_{m}}\right) V_{U_{2}, \ldots, U_{m}}^{*} .
$$

Using the trace function, we see that all the maps $\varphi_{j_{2}, \ldots, j_{m}}^{U_{2}, \ldots, U_{m}}$ have to be the same. Assume that this common map is equal to $\varphi$, which is either the identity map or the transposition map. By linearity, one can conclude that for any $A=A_{2} \otimes \cdots \otimes A_{m} \in H_{n_{2} \cdots n_{m}}$ and $B \in H_{n_{1}}$,

$$
\phi\left(B \otimes A_{2} \otimes \cdots \otimes A_{m}\right)=\varphi(B) \otimes \psi\left(A_{2} \otimes \cdots \otimes A_{m}\right)
$$

for some $\psi(A)=\psi_{1}\left(A_{2} \otimes \cdots \otimes A_{m}\right) \in H_{n_{2} \cdots n_{m}}$, where $\psi(A)$ depends on $A$ only. Note that $\psi: H_{n_{2} \cdots n_{m}} \rightarrow H_{n_{2} \cdots n_{m}}$ is a linear map and $\sigma(\psi(A))=\sigma(A)$ for all $A \in H_{n_{2} \cdots n_{m}}$. Hence, by induction hypothesis, $\phi$ has the form (7), as desired. The proof is completed. 
Theorem 3.5. A linear map $\phi: H_{n_{1} \cdots n_{m}} \rightarrow H_{n_{1} \cdots n_{m}}$ satisfies

$$
r\left(\phi\left(A_{1} \otimes \cdots \otimes A_{m}\right)\right)=r\left(A_{1} \otimes \cdots \otimes A_{m}\right)
$$

for all $A_{1} \otimes \cdots \otimes A_{m} \in H_{n_{1} \cdots n_{m}}$ if and only if there is a unitary $U \in M_{n_{1} \cdots n_{m}}$ and $\lambda \in\{-1,1\}$ such that

$$
\phi\left(A_{1} \otimes \cdots \otimes A_{m}\right)=\lambda U\left(\varphi_{1}\left(A_{1}\right) \otimes \cdots \otimes \varphi_{m}\left(A_{m}\right)\right) U^{*}
$$

where $\varphi_{j}$ is the identity map or the transposition map $X \mapsto X^{t}$ for $j \in\{1, \ldots, m\}$.

Proof. The sufficiency part is clear. To prove the converse, by a similar argument as in Theorem 3.3 , we can show that $\phi\left(E_{j_{1} j_{1}} \otimes \cdots \otimes E_{j_{m} j_{m}}\right)$ has an eigenvalue in $\{1,-1\}$ for any index set $\left(j_{1}, \ldots, j_{m}\right)$, where $1 \leq j_{p} \leq n_{p}$ with $1 \leq p \leq m$. Next, one can show that for any orthogonal diagonal matrix $D_{1} \in H_{n_{1}}, \phi\left(D_{1} \otimes E_{j_{2} j_{2}} \otimes \cdots \otimes E_{j_{m} j_{m}}\right)$ has at least $n_{1}$ eigenvalues lying in $\{1,-1\}$. Furthermore, for any orthogonal diagonal matrices $D_{1} \in H_{n_{1}}$ and $D_{2} \in H_{n_{2}}, \phi\left(D_{1} \otimes D_{2} \otimes E_{j_{3} j_{3}} \otimes \cdots \otimes E_{j_{m} j_{m}}\right)$ has at least $n_{1} n_{2}$ eigenvalues lying in $\{1,-1\}$. Recurrently, one can show that for any orthogonal diagonal $D_{p} \in H_{n_{p}}$ with $1 \leq p \leq m, \phi\left(D_{1} \otimes D_{2} \otimes \cdots \otimes D_{m}\right)$ has $n_{1} n_{2} \cdots n_{m}$ eigenvalues lying in $\{1,-1\}$. This is possible only when $\phi\left(E_{j_{1} j_{1}} \otimes \cdots \otimes E_{j_{m} j_{m}}\right)$ is rank one and for any distinct index sets $\left(j_{1}, \ldots, j_{m}\right)$ and $\left(k_{1}, \ldots, k_{m}\right), \phi\left(E_{j_{1} j_{1}} \otimes \cdots \otimes E_{j_{m} j_{m}}\right)$ and $\phi\left(E_{k_{1} k_{1}} \otimes \cdots \otimes E_{k_{m} k_{m}}\right)$ have disjoint support. Therefore, there is a unitary matrix $W \in M_{n_{1} \cdots n_{m}}$ and $\mu_{j_{1}, \ldots, j_{m}} \in\{1,-1\}$ such that

$$
\phi\left(E_{j_{1} j_{1}} \otimes \cdots \otimes E_{j_{m} j_{m}}\right)=\mu_{j_{1}, \ldots, j_{m}} W\left(E_{j_{1} j_{1}} \otimes \cdots \otimes E_{j_{m} j_{m}}\right) W^{*} .
$$

Suppose $P_{j_{2}, \ldots, j_{m}}$ are diagonal orthogonal matrices such that

$$
\phi\left(I_{n_{1}} \otimes E_{j_{2} j_{2}} \otimes \cdots \otimes E_{j_{m} j_{m}}\right)=W\left(P_{j_{2}, \ldots, j_{m}} \otimes E_{j_{2} j_{2}} \otimes \cdots \otimes E_{j_{m} j_{m}}\right) W^{*} .
$$

Since every rank one matrix $R \in H_{n_{1}}$ can be expressed as $U E_{11} U^{*}$ for some unitary $U \in M_{n_{1}}$, using the same argument as above, one can show that $\phi\left(R \otimes E_{j_{2} j_{2}} \otimes \cdots \otimes E_{j_{m} j_{m}}\right)$ has rank one with spectral radius 1 for all $1 \leq j_{p} \leq n_{p}$ with $2 \leq p \leq m$. By considering

$$
r\left(\phi\left(\left(R+t I_{n_{1}}\right) \otimes E_{j_{2} j_{2}} \otimes \cdots \otimes E_{j_{m} j_{m}}\right)\right)=1+t \quad \text { for all } t>0,
$$

one can conclude that $\phi\left(R \otimes E_{j_{2} j_{2}} \otimes \cdots \otimes E_{j_{m} j_{m}}\right)=W\left(\psi_{j_{2}, \ldots, j_{m}}(R) \otimes E_{j_{2} j_{2}} \otimes \cdots \otimes E_{j_{m} j_{m}}\right) W^{*}$ and hence for any $B \in H_{n_{1}}$,

$$
\phi\left(B \otimes E_{j_{2} j_{2}} \otimes \cdots \otimes E_{j_{m} j_{m}}\right)=W\left(\psi_{j_{2}, \ldots, j_{m}}(B) \otimes E_{j_{2} j_{2}} \otimes \cdots \otimes E_{j_{m} j_{m}}\right) W^{*} .
$$

Clearly, $\psi_{j_{2}, \ldots, j_{m}}$ preserves spectral radius on $H_{n_{1}}$ and, hence, has the form

$$
B \mapsto \xi Y B Y^{*} \quad \text { or } \quad B \mapsto \xi Y B^{t} Y^{*}
$$

for some $\xi \in\{1,-1\}$ and unitary $Y \in M_{n_{1}}$. Then, one can see that the scalar $\mu_{j_{1}, \ldots, j_{m}}$ has to be independent of the first index $j_{1}$, i.e., $\mu_{j_{1}, j_{2} \ldots, j_{m}}=\mu_{j_{1}^{\prime}, j_{2}, \ldots, j_{m}}$ for any $1 \leq j_{1}, j_{1}^{\prime} \leq n_{1}$. Applying the same argument on the $p$ th subsystem for $p=2, \ldots, m$, one can deduce that $\mu_{j_{1}, \ldots, j_{m}}$ is independent of the $p$ th index $j_{p}$. Therefore, $\mu_{j_{1}, \ldots, j_{m}}=\mu_{k_{1}, \ldots, k_{m}}$ for any the index sets $\left(j_{1}, \ldots, j_{m}\right)$ and $\left(k_{1}, \ldots, k_{m}\right)$ and hence $\mu_{j_{1}, \ldots, j_{m}}=\mu$ is a constant. So

$$
\phi\left(E_{j_{1} j_{1}} \otimes \cdots \otimes E_{j_{m} j_{m}}\right)=\mu W\left(E_{j_{1} j_{1}} \otimes \cdots \otimes E_{j_{m} j_{m}}\right) W^{*} \quad \text { for all } \quad 1 \leq j_{p} \leq m \text { with } 1 \leq p \leq m .
$$

By the same argument, one can show that for any unitary $U_{p} \in M_{n_{p}}$ with $1 \leq p \leq m$,

$$
\phi\left(U_{1} E_{j_{1} j_{1}} U_{1}^{*} \otimes \cdots \otimes U_{m} E_{j_{m} j_{m}} U_{m}^{*}\right)=\mu_{U_{1}, \ldots, U_{m}} W_{U_{1}, \ldots, U_{m}}\left(E_{j_{1} j_{1}} \otimes \cdots \otimes E_{j_{m} j_{m}}\right) W_{U_{1}, \ldots, U_{m}}^{*}
$$


for all $1 \leq j_{p} \leq n_{p}$ with $1 \leq p \leq m$. Here the scalar $\mu_{U_{1}, \ldots, U_{m}} \in\{1,-1\}$ and the unitary matrix $W_{U_{1}, \ldots, U_{m}} \in M_{n_{1} \cdots n_{m}}$ depend on $U_{1}, \ldots, U_{m}$ only. Furthermore, summing up for all the indices $j_{1}, \ldots, j_{m}$ yields $\phi\left(I_{n_{1} \cdots n_{m}}\right)=\mu_{U_{1}, \ldots, U_{m}} I_{n_{1} \cdots n_{m}}$. So $\mu_{U_{1}, \ldots, U_{m}}=\mu_{I_{n_{1}, \ldots, I_{n_{m}}}}=\mu$ is independent of the choice of $U_{1}, \ldots, U_{m}$. Without loss of generality, we may assume that $\mu=1$. Then by linearity, $\sigma\left(\phi\left(A_{1} \otimes \cdots \otimes A_{m}\right)\right)=\sigma\left(A_{1} \otimes \cdots \otimes A_{m}\right)$ for all $A_{1} \otimes \cdots \otimes A_{m} \in H_{n_{1}} \otimes \cdots \otimes H_{n_{m}}$, and the result follows from Theorem 3.4 .

3.3. Additional remarks and results. Several remarks concerning our results in the last two subsections are in order.

First, in all previous study of linear preservers involving tensor product spaces, one always imposed the assumption that the preservers send tensor states to tensor states. As a result, the structure of the preservers have the form

$$
A \otimes B \mapsto \psi_{1}(A) \otimes \psi_{2}(B) \quad \text { or } \quad A \otimes B \mapsto \psi_{2}(B) \otimes \psi_{1}(A) .
$$

In our case, we do not assume that the preservers send tensor states to tensor states. Nevertheless, our results show that up to a unitary similarity, we still have the form (9).

Second, we characterize linear operators $\phi$ such that $A \otimes B$ and $\phi(A \otimes B)$ have the same spectrum (respectively, spectral radius). The resulting map may not preserve the spectrum (respectively, spectral radius) of a general matrix $C \in H_{m n}$. For example, if $C=E_{11} \otimes E_{11}+E_{22} \otimes E_{22}+E_{12} \otimes$ $E_{12}+E_{21} \otimes E_{21}$, then the map $\phi$ of the form $A \otimes B \mapsto A \otimes B^{t}$ for tensor states will preserve the spectral radius (and spectrum) of tensor states, but $\phi(C)$ and $C$ will not have the same spectral radius (and spectrum). One can easily extend the above observation to the following.

Theorem 3.6. Suppose $\phi: H_{n_{1} \cdots n_{m}} \rightarrow H_{n_{1} \cdots n_{m}}$ is linear such that $r(\phi(C))=r(C)$ (respectively, $\sigma(\phi(C))=\sigma(C))$ for all $C=A_{1} \otimes \cdots \otimes A_{m}$ with $A_{j} \in H_{n_{j}}, j=1, \ldots, m$, and for $C$ obtained from $I_{n_{1}} \otimes \cdots \otimes I_{n_{m}}$ by replacing $I_{n_{i}} \otimes I_{n_{i+1}}$ with $E_{11} \otimes E_{11}+E_{22} \otimes E_{22}+E_{12} \otimes E_{12}+E_{21} \otimes E_{21}$, $i=1, \ldots, m-1$. Then there are a unitary $U$ and $\xi \in\{1,-1\}$ (respectively, $\xi=1$ ) such that $\phi$ has the form

$$
X \mapsto \xi U X U^{*} \quad \text { or } \quad X \mapsto \xi U X^{t} U^{*} .
$$

Third, one may consider affine maps $\psi$ on the set of density matrices in $H_{N}=H_{n_{1}} \otimes \cdots \otimes H_{n_{m}}$ instead of linear maps on $H_{N}$. One may extend an affine map on density matrices in $H_{N}$ in the standard way, namely, define for any positive semi-definite matrix $C, \phi(t C)=t \phi(C)$, and $\phi(C)=\psi(C)$ if $\operatorname{tr} C=1$. Then use the fact that every $X \in H_{N}$ is a difference of two positive semi-definite $C_{1}$ and $C_{2}$, and that $\phi\left(C_{1}\right)-\phi\left(C_{2}\right)=\phi\left(D_{1}\right)-\phi\left(D_{2}\right)$ if $C_{1}-C_{2}=D_{1}-D_{2}$.

Finally, it is interesting to study (real or complex) linear maps $\phi: M_{m} \otimes M_{n} \rightarrow M_{m} \otimes M_{n}$ such that $A \otimes B$ and $\phi(A \otimes B)$ always have the same spectrum (respectively, spectral radius).

\section{Acknowledgment}

This research was supported by a Hong Kong RCG grant PolyU 502910 with Sze as PI and Li as co-PI. The grant also supported the post-doctoral fellowship of Huang and the visit of Fošner to the Hong Kong Polytechnic University in the spring of 2011. She gratefully acknowledged the support and kind hospitality from the host university. Li was also supported by a USA NSF grant; this research was done when he was a visiting professor of the University of Hong Kong in the 
spring of 2012; furthermore, he is an honorary professor of Taiyuan University of Technology (100 Talent Program scholar), and an honorary professor of the Shanghai University.

\section{REFERENCES}

[1] M. Brešar, M.A. Chebotar, W.S. Martindale III, Functional identities. Frontiers in Mathematics. Birkhuser Verlag, Basel, 2007.

[2] J.T. Chan, C.K. Li, and N.S. Sze, Mappings preserving spectra of product of matrices, Proc. Amer. Math. Soc., 135(2007) 977-986.

[3] D. Chruscinski, A. Kossakowski, and G. Sarbicki, Spectral conditions for entanglement witnesses vs. bound entanglement, Phys. Rev A 80 (2009), 042314.

[4] J. Dieudonné, Sur une gén eralisation du groupe orthogonal á quatre variables, Arch. Math. 1 (1949), 282287.

[5] S. Friedland, C.K. Li, Y.T. Poon and N.S. Sze, The automorphism group of separable states in quantum information theory, Journal of Mathematical Physics 52 (2011), 042203.

[6] G. Frobenius, Uber die Darstellung der endlichen Gruppen durch Linear Substitutionen, Sitzungsber Deutsch. Akad. Wiss. Berlin (1897) 994-1015.

[7] L. Gurvits, Classical deterministic complexity of Edmonds problem and quantum entanglement, in Proceedings of the 35th ACM Symposium on Theory of Computing, ACM Press, New York, 2003.

[8] J.C. Hou et al., Private communication.

[9] A.A. Jafarian and A.R. Sourour, Spectrum-preserving linear maps. J. Funct. Anal. 66 (1986), 255-261.

[10] N. Johnston, Characterizing Operations Preserving Separability Measures via Linear Preserver Problems, Linear and Multilinear Algebra 59 (2011), 1171-1187.

[11] N. Johnston and D.W. Kribs, A family of norms with applications in quantum information theory, J. Math. Phys. 51 (2010), 082202.

[12] N. Johnston and D.W. Kribs, A family of norms with applications in quantum information theory II, Quant. Inf. Comp. 11 (2011), 104-123.

[13] N. Johnston, D.W. Kribs, V.I. Paulsen, and R. Pereira, Minimal and maximal operator spaces and operator systems in entanglement theory, J. Funct. Anal. 260 (2011), 2407-2423.

[14] C.K. Li, Linear operaters preserving the numerical radius of matrices, Proc. Amer. Math. Soc. 99 (1987), 601-608.

[15] C.K. Li and S. Pierce, Linear preserver problems, Amer. Math. Monthly 108 (2001), 591-605.

[16] C.K. Li, L. Plevnik and P. Šemrl, Preservers of matrix pairs with a fixed inner product value, to appear in Operators and Matrices, http://files.ele-math.com/preprints/oam-0491-pre.pdf

[17] C.K. Li, Y.T. Poon and N.S. Sze, Linear preservers of Tensor product of Unitary Orbits, and Product Numerical Range, Linear Algebra Appl., to appear.

[18] M.H. Lim, Additive preservers of tensor product of rank one Hermitian matrices, Electronic J. Linear Algebra, to appear.

[19] M. Marcus and B. Moyls, Linear transformations on algebras of matrices, Canad. J. Math. 11 (1959) 61-66.

[20] L. Molnár, An algebraic approach to Wigner's unitary-antiunitary theorem, J. Austral. Math. Soc. Ser. A 65 (1998), 354-369.

[21] L. Molnár, Characterizations of the automorphisms of Hilbert space effect algebras, Comm. Math. Phys. 223 (2001), 437-450.

[22] L. Molnár, Order-automorphisms of the set of bounded observables, J. Math. Phys. 42 (2001), 5904-5909.

[23] L. Molnár, Conditionally multiplicative maps on the set of all bounded observables preserving compatibility, Linear Algebra Appl. 349 (2002), 197-201.

[24] L. Molnár, Orthogonality preserving transformations on indefinite inner product spaces: generalization of Uhlhorn's version of Wigner's theorem, J. Funct. Anal. 194 (2002), 248-262.

[25] L. Molnár, Preservers on Hilbert space effects, Linear Algebra Appl. 370 (2003), 287-300.

[26] L. Molnár, L. Selected preserver problems on algebraic structures of linear operators and on function spaces, Lecture Notes in Mathematics, 1895. Springer-Verlag, Berlin, 2007. 
[27] L. Molnár and M. Barczy, Linear maps on the space of all bounded observables preserving maximal deviation, J. Funct. Anal. 205 (2003), 380-400.

[28] L. Pankowski, M. Piani, M. Horodecki, and P. Horodecki, A few steps more towards NPT bound entanglement, IEEE Trans. Inf. Theory 56 (2010), 4085-4100.

[29] H. A. Robinson, Quadratic Forms on Symmetry Classes of Tensors, Linear and Multilinear Algebra 4 (1977), 233-241.

[30] A. SaiToh, R. Rahimi, and M. Nakahara, Mathematical framework for detection and quantification of nonclassical correlation, Quantum Inf. Comput. 11 (2011), 0167-0180.

[31] A. SaiToh, R. Rahimi, M. Nakahara, Limitation for linear maps in a class for detection and quantification of bipartite nonclassical correlation, preprint. arXiv:1012.5718

[32] U. Uhlhorn, Representation of symmetry transformations in quantum mechanics, Ark. Fysik 23 (1963), 307-340.

[33] Z.X. Wan, Geometry of matrices. In memory of Professor L. K. Hua (1910-1985), World Scientific Publishing Co., Inc., River Edge, NJ, 1996.

Ajda Fošner, Faculty of Management, University of Primorska, Cankarjeva 5, SI-6104 Koper, SLOVENIA

E-mail address: ajda.fosner@fm-kp.si

Zejun Haung, Department of Applied Mathematics, The Hong Kong Polytechnic University, Hung HOM, HONG KONG

E-mail address: huangzejun@yahoo.cn

Chi-Kwong Li, Department of Mathematics, College of William and Mary, Williamsburg, VA 23187 , USA; Department of Mathematics, University of Hong Kong, Pokfulam, Hong Kong

E-mail address: ckli@math.wm.edu

Nung-Sing Sze, Department of Applied Mathematics, The Hong Kong Polytechnic University, Hung Hom, HONG KONG

E-mail address: raymond.sze@polyu.edu.hk 